AMERICAN JOURNAL OF SCIENTIFIC AND INDUSTRIAL RESEARCH

(C) 2010, Science Huß, http://www.scihub.org/AJSIR

ISSN: 2153-649X doi:10.5251/ajsir.2010.1.3.516.526

\title{
Using mathematical model to depict the immune response to Hepatitis B virus infection
}

\author{
Dontwi, I. K., Frempong, N.K. and Wiah, E. N. \\ Department of Mathematics, KNUST, Kumasi-Ghana \\ ABSTRACT
}

Hepatitis B is potentially a life-threatening liver infection caused by the Hepatitis B Virus (HBV) and is a major global health problem. A simplified mathematical model of immune responds to HBV infection is presented. This focuses on the control of the infection by the interferons, the innate and adaptive immunity. Parameter values for the model are obtained. We perform sensitivity analysis on the model parameters, to account for the variability and speed of adaptation.We demonstrate that for small initial viral load the disease progresses through an asymptotic course, for intermediate value it takes a typical course with constant duration and severity of infection but variable onset, and for large initial viral load the disease become severe.

Keywords: Immune system, Immunity, Immune response, Hepatitis B.

\section{INTRODUCTION}

Hepatitis B is a liver infection caused by the Hepatitis $B$ Virus (HBV). It can be a serious disease that can lead to cirrhosis (scarring of the liver) and/or liver cancer. Most people who get the disease recover from it and can never get it again, that is they get permanent immunity. However, about $10 \%$ of people who get hepatitis $B$ will go on to have chronic infection (long-term infection) and can pass it on to others (be carriers). The younger a person is when infected, the more likely he or she is to go on to have chronic infection and to develop serious liver disease.

The HBV is spread by direct contact with blood, semen, vaginal and other body fluids of an infected person. Sharing needles to inject drugs or having sex with an infected person can spread the virus. Health care workers who get struck with contaminated needles can also get infected if they are not protected by immunity, such as by immunization or vaccination. Pregnant women who have the virus in their blood can pass it on to their babies at birth (vertical transmission). The virus can also spread by sharing personal items such as toothbrush, razor, or anything else that may have blood on it.

In this paper we construct a simplified, biologically justified, mathematical model of the dynamics of HBV infection and the human immune response to such infection. We do not strive to obtain a detailed model accounting for all known components of the immune system and their interactions. Rather, the focus is on three important components of the immune response; the interferon and cellular components of innate immunity and the adaptive immunity. All of these have the same goal of limiting the concentration of the virus and the damage to the system. They achieve this goal using different strategies: interferon immunity by removing the Isubstrate" that the virus needs for reproduction (i.e., the healthy cells), cellular immunity by removing the source of new viruses (i.e., the infected cells), and adaptive immunity by lowering the effective concentration of the virus.

\subsection{The Immune Response to the Hepatitis B Virus in Humans}

Patients infected with HBV may develop one of two types of anti-HBV immune responses. The first is an effective anti-viral response that suppresses viral growth as the result of both non-specifics (innate) as well as specific (adaptive) immunity. After early actions of natural killer (NK) cells, NK T cells and antiviral cytokines, individuals with acute self-limited HBV infection mount a vigorous polyclonal and multispecific Th and CTL response to epitopes within the HBV-envelope, nucleocapsid and polymerase proteins. The peptides are presented to the T cells by professional antigen-presenting cells (APC) and on the surface of infected hepatocytes in the context of a MHC class I molecule. The response is readily detectable in peripheral blood (Maini et al., 2000; Rehermann et al., 1996; Livingston et al., 1997; Guidotti et al.,1994). It often coincides with an elevation of serum alanine-aminotransferase levels and precedes clearance of HBe and HBs (surface) antigens and the development of neutralizing antibodies. Upon recognition of viral peptides CTL acquire the capacity to either cure HBV-infected cells via a non-cytopathic, cytokine mediated inhibition of HBV replication, or to kill them via perforin-Fas ligand and TNF _-mediated death pathways (Rehermann et 
al., 2000; Ganem et al., 1982; Guidotti et al., 1999; Heise et al., 1999). Both effectors functions have been observed during resolution of acute HBV and this type 1 T-cell (Th1) response persists even after clinical recovery (Guidotti et al., 2000). In contrast, the second type of anti-HBV immune response is an ineffective one. The HBV-specific immune response is weak, antigenically restricted or undetectable in the blood of chronically infected patients, although individual HBV-specific T-cell clones have been isolated and expended from liver biopsies (Barnaba et al., 1989; Ferrari et al., 1987). As HBV is considered a non-cytopathic virus and the degree of intrahepatic inammatory leukocytic infiltrate is regarded as the histological hallmark of the severity of chronic hepatitis $B$, it has been postulated that the HBV-specific immune response is too weak to eliminate HBV from all infected hepatocytes, but sufficiently strong to continuously destroy HBVinfected hepatocytes and to induce chronic inammatory liver disease in persistently infected individuals.

Mathematical Syntheses: For the model of human immune response against HBV infection, consider a simplified model of population-dynamics type which consists of the following interactions (see Figure 1)
(Baris et al., 1998): The liver cells are assumed to be in one of four possible states: healthy $(H)$, infected $(I)$, dead (D), or resistant (R) to infection. The total number of liver cells (i.e., $H+I+D+R$ ) is assumed constant. The virus particles $(V)$ interact with healthy cells and infect them. Infected cells release new virus particles upon their death. Proliferation of healthy cells causes regeneration and decrease in the proportion of dead cells. Dead cells stimulate the activation of APC (M).

APC stimulate the production of interferon $\alpha$ and $\beta$ $(F)$ that interact with healthy cells and convert them to a resistant state. APC also stimulate the proliferation of effector cells (E) that destroy infected cells. Finally, they stimulate the production of plasma cells $(P)$ which, in turn, produce antibodies (A) that neutralize (kills) virus. This neutralization is modulated by the antigenic compatibility (S) between virus and antibodies currently produced by the organism. S quantities the affinity between antibodies and virus. These interactions are used in the construction of a system of 10-dimensional ordinary differential equations describing the dynamics of the main variables, which correspond to the components of the immune response shown in figure 1 below.

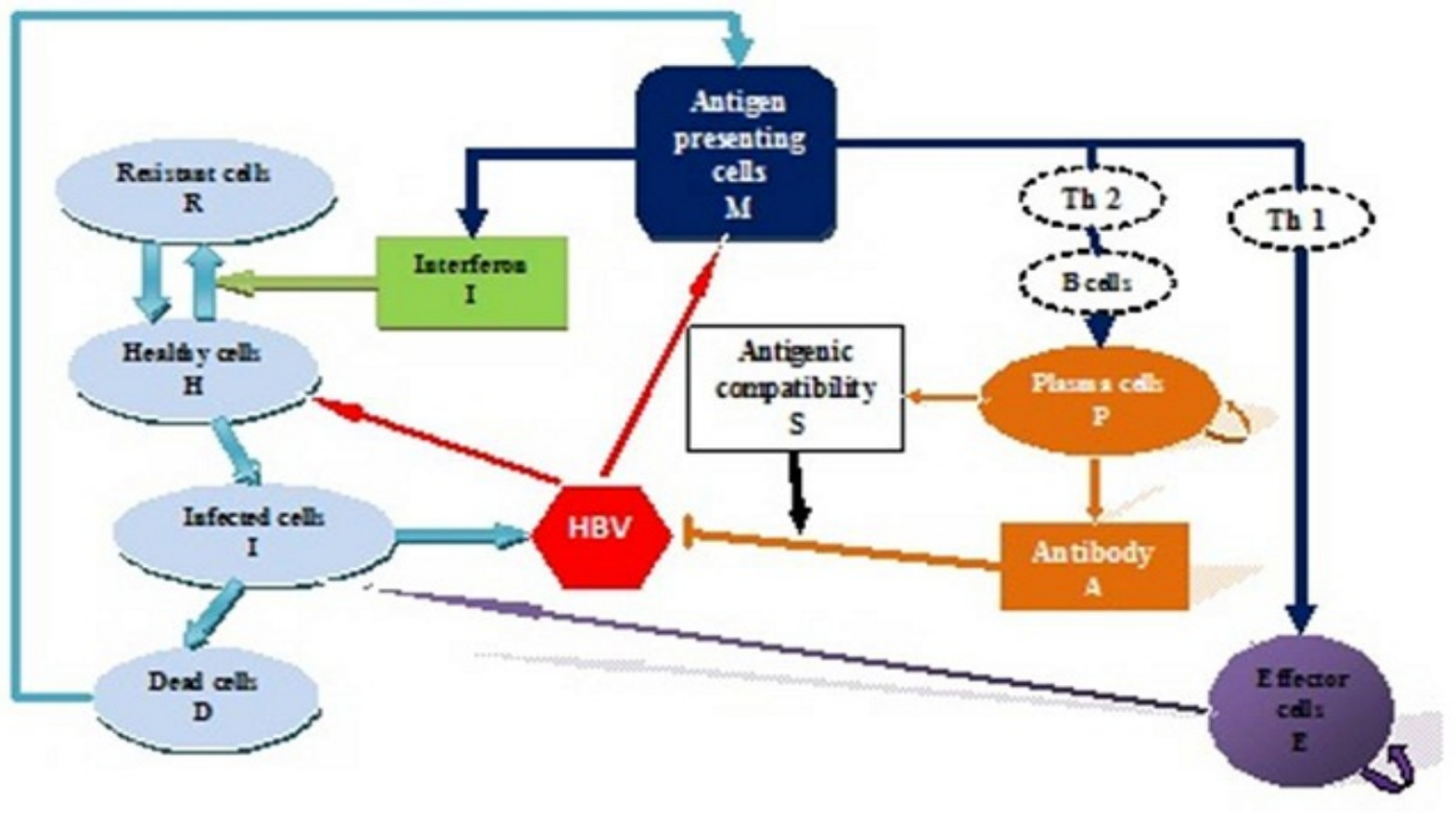

Fig 1: Schematic representation of interactions included in the model. 
$\frac{d V}{d t}=\alpha I-\varepsilon S A V-\theta H V-\lambda V$

$\frac{d H}{d t}=\pi D(H+R)+\sigma R-\varphi V H-\beta F H$

$\frac{d I}{d t}=\varphi V H-\mu E I-\tau I$

$\frac{d M}{d t}=(\vartheta D+\chi V)(1-M)-\gamma M$

$\frac{d F}{d t}=\psi M+\delta I-\eta H F-\xi F$

$\frac{d R}{d t}=\beta F H-\sigma R$

$\frac{d E}{d t}=\in M E-\rho I E+\omega(1-E)$

$\frac{d P}{d t}=\phi M P+\kappa(1-P)$

$\frac{d A}{d t}=\tilde{\mathrm{n}} P-\varepsilon S A V-\zeta A$

$\frac{d S}{d t}=r P(1-S)$

No differential equation is needed for the proportion of dead cells (D) which is given by

$D=1-H-R-I$

The variable $D$ serves as a marker for tissue damage (Hayden et al., 1998) and an indicator of the severity of disease. The system (1)-(11) is nondimensionless. The nondimensionless here means that, since we do not know the actual values of the variable, we assume these values to be in proportions.

The interactions are based on clonal selection theory, mass-action kinetics, characteristics of interactions and the birth-death balances of populations of cells and molecules.

We note that a number of assumptions in the model are strong simplifications of our knowledge of immune physiology.

- We assume that the system is healthy, thus free of all infection such as HIV, Influenza, etc. We now introduce HBV and looked at the dynamics.

- The populations of cells and virions are assumed to be uniformly distributed over the system at all times.

- It is also assumed that time rate of change of any model variable is determined by the present value of all variables.

- We do not account for the intermediate steps in the production of effector cells and plasma cells such as Th1 and Th2 helper cells and B-cells.
(2)

- We do not consider time delays in the reproduction of cellular components.

Simulations: The simulations were conducted (B)ing MATLAB's ode15s method with a Relative Tolerance of $2 \mathrm{e}^{-29}$ and an Absolute Tolerance of $1 \mathrm{e}^{-}$ (14). The Relative Tolerance property specifies the (allowable error tolerance relative to the state vector at each simulation step, while the Absolute (Folerance property specifies the largest allowable absolute error at any step in simulation. Thus at (higher state values, convergence is determined by Relative Tolerance. As the state values approach Zero, convergence is controlled by Absolute Jolerance. The choice of values for Relative Tolerance and Absolute Tolerance will vary (depending on the problem.

We take or derive all parameters from the literature; the time courses of variables were obtained by numerical integration using parameters provided in Table 1. Model parameters were adjusted so that the response of the naive host to the standard initial conditions satisfies the following criteria, extracted from available experimental and clinical data (Bocharov et al., 1994):

\section{RESULTS}

Standard Behavior: The standard behavior describes the course of infection in a naive host. We assume that initially the host has no dead, infected or resistant cells, no interferon molecules, and no activated APC (i.e., $\mathrm{H}(0)=0.59, \mathrm{I}(0)=0$, $M(0)=0, F(0)=0, R(0)=0)$. The initial levels of effectors, plasma cells, and antibodies are assumed to be at this values (i.e., $\mathrm{E}(0)=0.1, \mathrm{P}(0)=0.1, \mathrm{~A}(0)$ $=0.1$ ). In a naive host, we assume that $S(0)=0.1$ which corresponds to a relatively low compatibility with the virus strain, that may have resulted from previous exposure to HBV and subsequent genetic drift. In the typical course of acute HBV Infection, the initial concentration of aerosol delivered virus particles that the host receives is about 106 particle per $\mathrm{ml}$ on day 0 , corresponding to $\mathrm{V}(0)=0.01$ in our dimensionless system.

Interferon response comes into play once the virus peaks at day 3 making most of the cells resistant to infection. Interferon level is increased by 280 fold peaking approximately at day 3 . Plasma cells are produced after 4 days peaking at 7 days, before virus-specific antibodies are detectable, in accord with empirical observations (Ada et al., 1986). 
Antibody production by plasma cells begins at day 4. There is $11.8 \times 10^{8}$ fold increase in the amount of antibodies when the adaptive immune response comes into play to remove all viral particles and generate immune memory. Furthermore, antigenic compatibility is increasing monotonically starting right after when the adaptive immunity is activated (after day 3 ) and the antibodies are capable of inhibiting viral particles with $80 \%$ probability after 20 day of infection (Tamura and Kutara, 2004).

Table 1: Model parameters used for the modeling

\begin{tabular}{|c|c|c|c|}
\hline Parameters & Values & Description & Source \\
\hline$\alpha$ & 100 & rate constant of HBV & Zdanov et al. 1969 \\
\hline$\varepsilon$ & 146.2 & rate constant of neutralization of HBV by antibodies & Bocharov et al. 1994 \\
\hline$\theta$ & 1.02 & rate constant of absorption of HBV by infected cells & Bocharov et al. 1994 \\
\hline$\lambda$ & 1.7 & rate constant of nonspecific HBV removal & Bocharov et al. 1994 \\
\hline$\pi$ & 4 & rate constant of regeneration & Keenan et al. 1982 \\
\hline$\sigma$ & 1 & rate constant of cell's virus resistance state decay & Marchuk et al. 1991 \\
\hline$\varphi$ & 0.34 & rate constant of cell infected by HVB & Marchuk et al. 1991 \\
\hline$\beta$ & 0.01 & rate constant of cells virus resistant state induction & Bocharov et al. 1994 \\
\hline$\mu$ & 0.066 & rate constant of infected cell that CTL damages & Bocharov et al. 1994 \\
\hline$\tau$ & 0.5 & rate constant of infection cell damage by cytopthicity of HBV & Zdanov et al. 1969 \\
\hline$\vartheta$ & 1 & $\begin{array}{l}\text { rate constant of stimulation of antigen presenting cell by dead } \\
\text { cell }\end{array}$ & Marchuk et al. 1991 \\
\hline$\chi$ & 0.037 & $\begin{array}{l}\text { rate constant of stimulation of antigen presenting cell by virus } \\
\text { particle }\end{array}$ & Marchuk et al. 1991 \\
\hline$\gamma$ & 1 & $\begin{array}{l}\text { rate constant of stimulated state lose of antigens presenting } \\
\text { cell }\end{array}$ & Marchuk et al. 1991 \\
\hline$\psi$ & 2500 & Interferon (IFN) production rate per APC & Bocharov et al. 1994 \\
\hline$\delta$ & 200 & Interferon (IFN) production rate per infected cell & Baris et al. 1998 \\
\hline$\eta$ & 17 & rate constant of cell that IFN bind & Bocharov et al. 1994 \\
\hline$\xi$ & 8 & rate constant of IFN's natural decay & Bocharov et al. 1994 \\
\hline$\epsilon$ & 8.3 & rate constant of stimulation of effector cell & Marchuk et al. 1991 \\
\hline$\rho$ & 2.72 & $\begin{array}{l}\text { rate constant of death of effector by lytic interaction with } \\
\text { infected cell }\end{array}$ & Bocharov et al. 1994 \\
\hline$\omega$ & 0.4 & rate constant of natural death of effector cell & Marchuk et al. 1991 \\
\hline$\phi$ & 11.5 & rate constant of plasma cell production & Marchuk et al. 1991 \\
\hline \multirow[t]{2}{*}{$\kappa$} & 0.4 & rate constant of natural death of plasma cell & Marchuk et al. 1991 \\
\hline & 0.43 & antibody production rate per plasma cell & Marchuk et al. 1991 \\
\hline$\zeta$ & 0.43 & rate constant of natural death of antibodies & Marchuk et al. 1991 \\
\hline$r$ & $3 e^{-1}$ & rate constant for $\mathrm{S}$ variable & Baris et al. 1998 \\
\hline
\end{tabular}


Am. J. Sci. Ind. Res., 2010, 1(3): 516-526

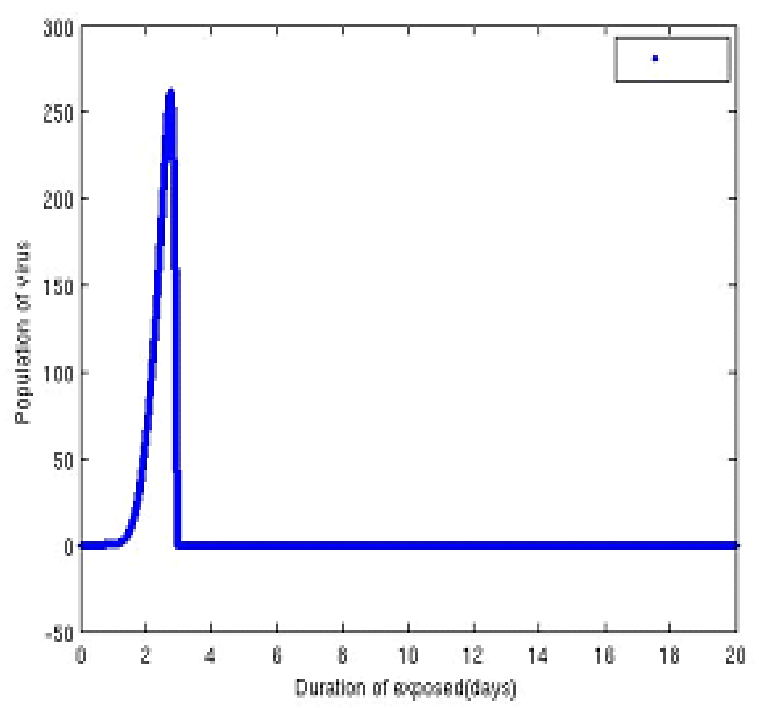

(A)

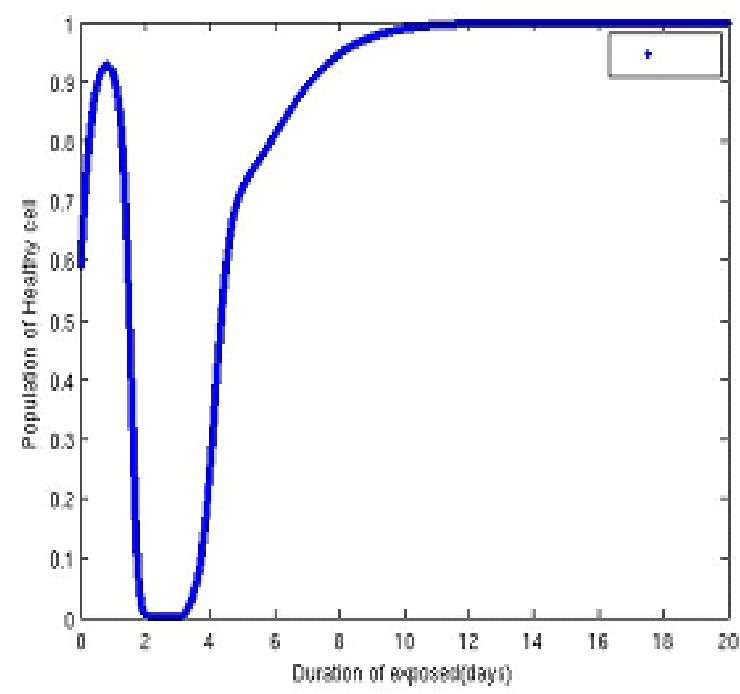

(B)

Fig 2: Virus and Healthy cells

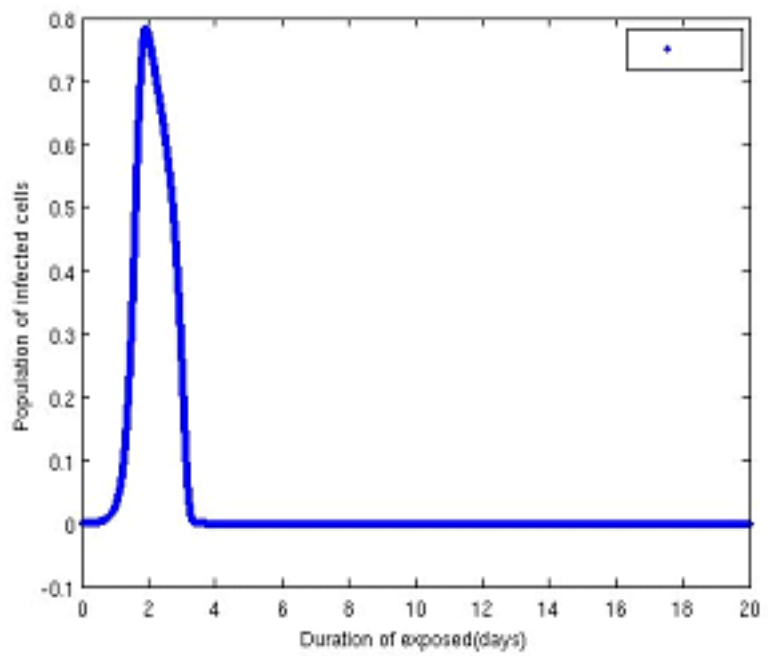

(c)

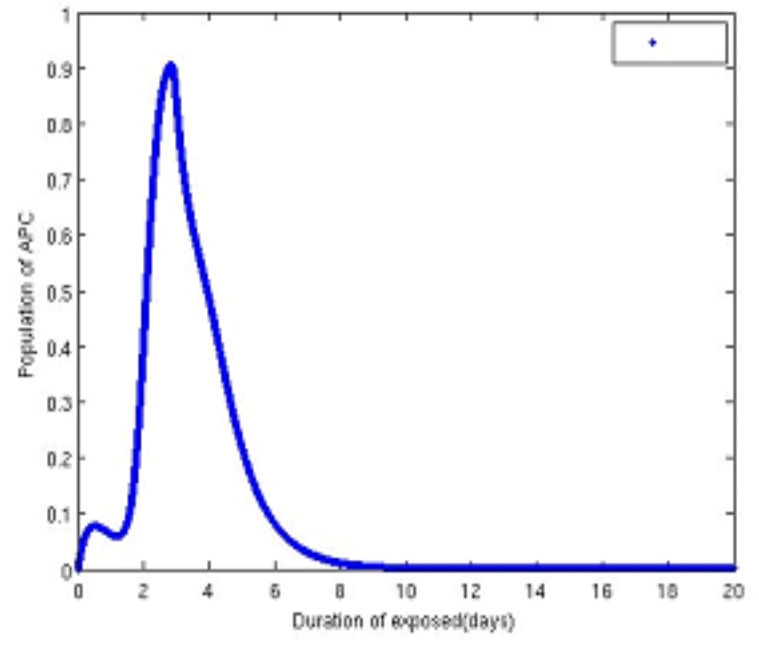

(D)

Fig 3: Infected cells and Activation antigen presenting cell (APC) 
Am. J. Sci. Ind. Res., 2010, 1(3): 516-526

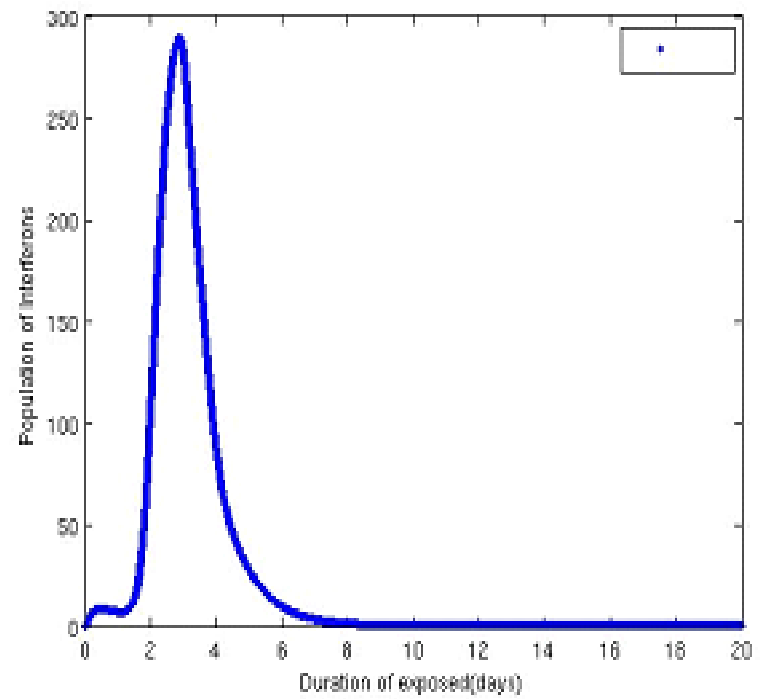

(E)

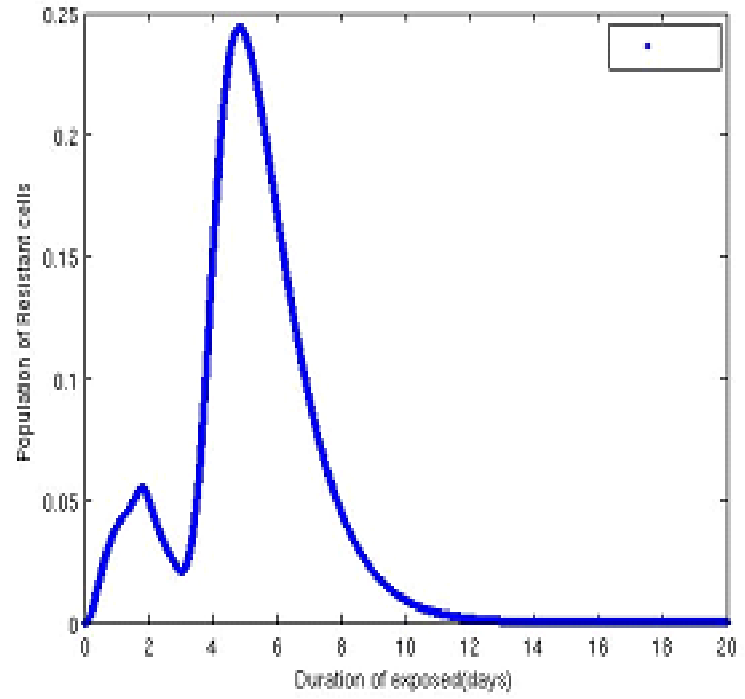

(F)

Fig 4: Interferons and Resistant cells

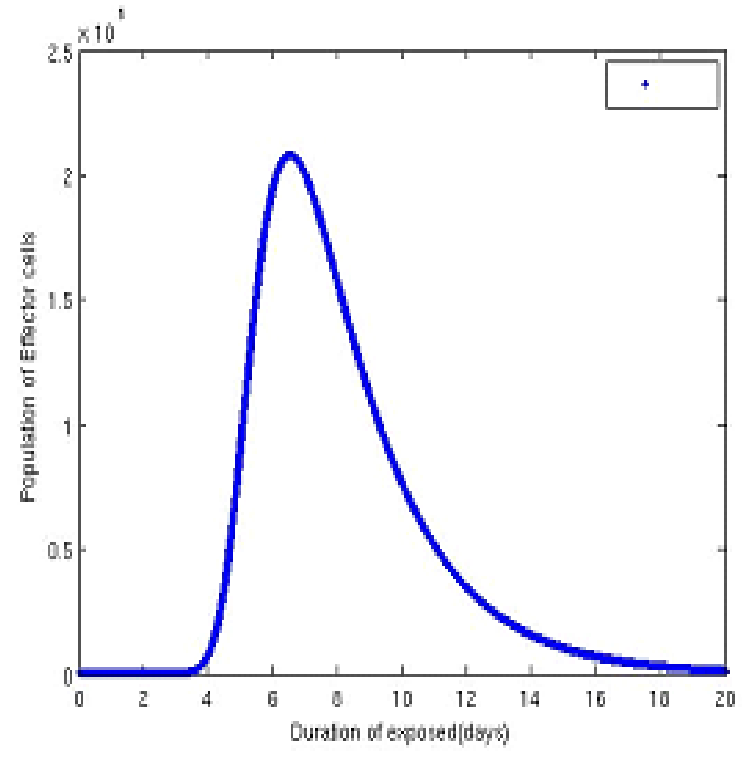

(G)

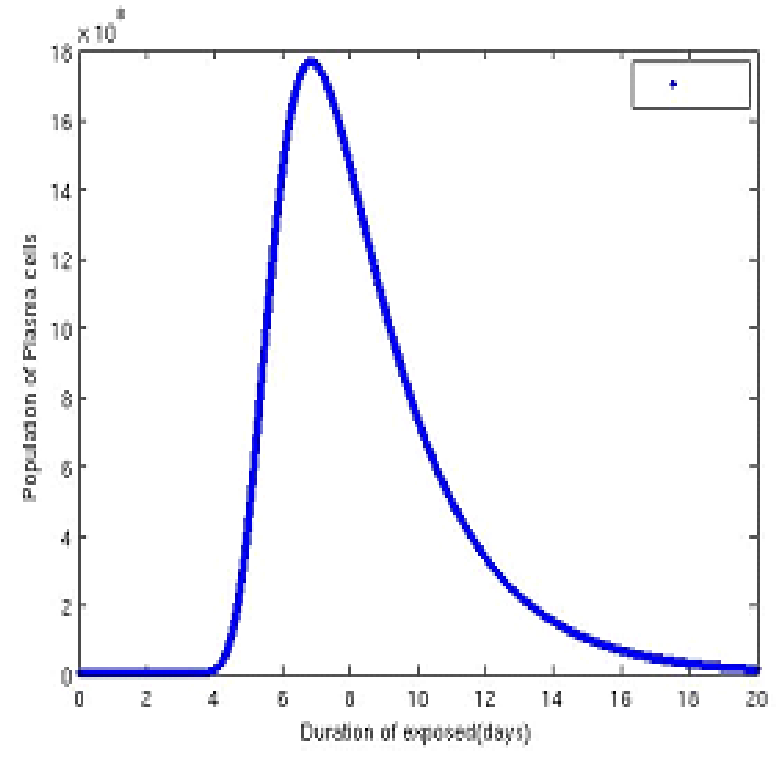

(H)

Fig 5: Effector cells and Plasma cells 
Am. J. Sci. Ind. Res., 2010, 1(3): 516-526

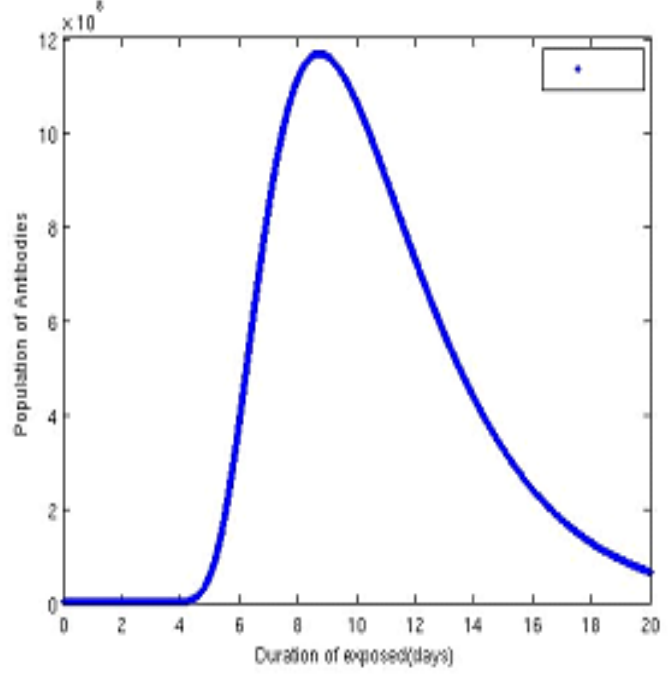

(I)

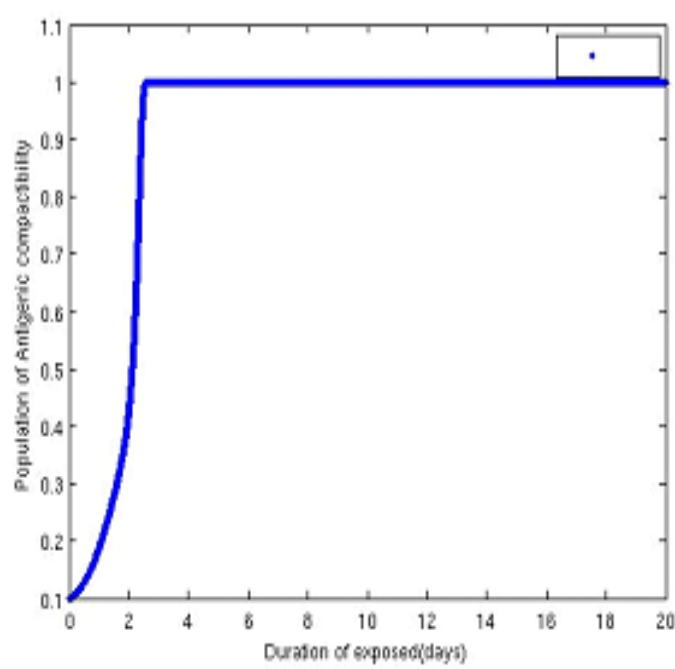

(J)

Fig 6: Antibodies and Antigenic compatibility

From fig. 1 (a). After a time lag of 2 days postinfection the virus begin to replicate in the system. From days 2 to 3 the production rate of viral particle by the infected cell break sharply to a maximum level of about 270 cells on day 3 . Between days 3 and 20 nearly all viral cells are neutralized by specific antibodies and some also die out. The model indicates that the number of viral particle declines from 270 cells on day 3 of the immune response. (b) The healthy cell gradually increases from day 0 of no infection. After a time lag of 1 day post-infection, the cells decline exponentially to 0 by days 2 and 3 when the healthy cell looses their resistance to infection. After day 3 the cell increases exponentially when there is a transition of the healthy cells into resistant cells by the interferons.

In fig. 2 (c). After a time lag of 1 day post-infection the infected begin to replicate in the system. From days 1 to 3 the infected cells break sharply to a maximum level of about 0.8 cells on day 2 . Between days 3 and 20 nearly all infected cells are neutralized by specific antibodies and some also die out. The model indicates that the number of viral particle declines from 0.8 cells on day 3 of the immune response. (d) The APC act as signal sensor. Upon the death of some infected cell, the APC is activated by day 1 and increase exponentially by day 1 and so doing it stimulate the interferon immunity, the innate and adaptive immunity when it peak at 0.9 by day 3 and then gradually decline exponentially by days 3 to 7 when the virus is in control.

From Figure $3(e)$ and $(f)$. The interferon is stimulated by APC by day 1 and increase exponentially by day 1 and peak at 280 by day 3 and then gradually decline exponentially by days 3 to 7 while doing this there is a transition of healthy cells to resistant cells which also increase exponentially by day 3 and peak at 0.24 by day 5 and then gradually decline exponentially by days 5 to 10 when the virus is in control.

From Figure 4 (g). Effector cells are stimulated by APC, appears around day 4 post-infection reach a peak of $2.1 \times 10^{5}$ by day 6 and decline exponentially. (h) Plasma cell are stimulated by APC, migrate into the infected tissues much faster than their proliferation, appears around day 4 post-infection reach a peak of $17.8 \times 10^{8}$ by day 7 and decline exponentially.

In Figure 5 (i). Antibodies appears around day 4 postinfection reach a peak of $11.5 \times 10^{8}$ by day 9 and then decline exponentially at rate of $\zeta$ (rate constant of natural death of antibodies). (j) This indicates a calculation with an increasing binding affinity of the antibody and the virus from days 0 and 2 .

Stability analysis: Stability of equilibrium points for disease-free environment 
We conduct linear stability on the equilibrium points for disease-free environment, $x_{d f e}$. System (1)-(10) yield steady states of the form $\left(V^{*}, H^{\star}, I^{*}, M^{*}, F^{*}, R^{*}\right.$, $\left.E^{\star}, P^{*}, A^{*}, S^{*}\right)$. The trivial equilibrium $(0,1,0,0,0,0$, $0,0,0,0)$ represents a disease-free environment. An analysis of the local stability of the trivial equilibrium enables us to identify condition under which a behavior of HBV infection can be established. The local stability may be determined from the eigenvalues of the Jacobian matrix. The Jacobian of the HBV model is in equation (12) below:

$$
J=\left(\begin{array}{llllllllll}
J_{1,1} & J_{1,2} & J_{1,3} & 0 & 0 & 0 & 0 & 0 & 0 & J_{1,10} \\
J_{2,1} & J_{2,2} & 0 & 0 & J_{2,5} & J_{2,6} & 0 & 0 & 0 & 0 \\
J_{3,1} & J_{3,2} & J_{3,3} & 0 & 0 & 0 & J_{3,7} & 0 & 0 & 0 \\
J_{4,1} & 0 & 0 & J_{4,4} & 0 & 0 & 0 & 0 & 0 & 0 \\
0 & J_{5,2} & J_{5,3} & J_{5,4} & J_{5,5} & 0 & 0 & 0 & 0 & 0 \\
0 & J_{6,2} & 0 & 0 & J_{6,5} & J_{6,6} & 0 & 0 & 0 & 0 \\
0 & 0 & J_{7,3} & J_{7,4} & 0 & 0 & J_{7,7} & 0 & 0 & 0 \\
0 & 0 & 0 & J_{8,4} & 0 & 0 & 0 & J_{8,8} & 0 & 0 \\
J_{9,1} & 0 & 0 & 0 & 0 & 0 & 0 & J_{9,8} & J_{9,9} & J_{9,10} \\
0 & 0 & 0 & 0 & 0 & 0 & 0 & J_{10,8} & 0 & J_{10,10}
\end{array}\right)
$$

With

$$
\begin{aligned}
& J_{1,1}=-\varepsilon S A-\theta \quad H-\lambda \\
& J_{1,2}=-\theta \mathrm{V} \\
& J_{1,3}=\alpha \\
& J_{1,10}=-\varepsilon A V \\
& J_{2,1}=-\varphi H \\
& J_{2,2}=\pi D-\varphi-\beta F \\
& J_{2,5}=-\beta H \\
& J_{2,6}=\pi D+\sigma \\
& J_{3,1}=\varphi H \\
& J_{3,2}=\varphi V \\
& J_{3,3}=-\mu E-\tau \\
& J_{3,7}=-\mu I \\
& J_{4,1}=\chi-\chi M \\
& J_{4,4}=\vartheta D-\chi V-\sigma \\
& J_{5,4}=\psi \\
& J_{5,5}=-\eta H-\xi
\end{aligned}
$$

$$
\begin{aligned}
& J_{6,2}=\beta F \\
& J_{6,5}=\beta H \\
& J_{6,6}=-\sigma \\
& J_{7,3}=-\rho E \\
& J_{7,4}=\text { ò } E \\
& J_{7,7}=\text { ò } M-\rho I-\omega \\
& J_{8,4}=\phi P \\
& J_{8,8}=\phi P-\kappa \\
& J_{9,1}=-\varepsilon S A \\
& J_{9,8}=\tilde{\mathbf{n}} \\
& J_{9,9}=-\varepsilon S V-\zeta \\
& J_{9,10}=-\varepsilon A V \\
& J_{10,8}=r-r S \\
& J_{10,10}=-r P
\end{aligned}
$$

Suppose that $x^{1}, x^{2}, \cdots, x^{10}$ are the 10 solutions of the $x^{\prime}=A(t) x$, where $\mathrm{A}$ is a 10 by 10 matrix. Then determinant

$$
\begin{aligned}
& W\left[x^{1}, x^{2}, \cdots, x^{10}\right]=\operatorname{det}\left[x^{1}(t), x^{2}(t), \cdots, x^{10}(t)\right] \\
& \text { is the wronskian of the } 10 \text { solutions } \\
& x^{1}(t), x^{2}(t), \cdots x^{10}(t) .
\end{aligned}
$$


Proposition (Stability of steady state) (Akinwande, 2009)

If the characteristics equation of a dynamical system is of the form

$$
\int_{0}^{\infty} e^{-\lambda t} W(t) d t=1
$$

where $\lambda$ is the eigenvalue, then the steady state of the system is unstable if

$$
\int_{0}^{\infty}|W(t)| d t>1
$$

while it is locally asymptotically stable if

$$
\int_{0}^{\infty}|W(t)| d t<1
$$

\section{Proof}

Let $\lambda=u \pm i v$, then equation (13) gives the pair of equations:

$$
\int_{0}^{\infty} e^{-u t} W(t) \cos v t d t=1
$$

and

$$
\int_{0}^{\infty} e^{-u t} W(t) \sin v t d t=0
$$

From stability theories, a necessary and sufficient condition for local asymptotic stability is for the real part of the eigenvalue to be in the negative half plane, i.e. $\operatorname{Re} \lambda=u<0$. Now, we note that the function

$$
g(u)=\int_{0}^{\infty} e^{-u t}|W(t)| d t
$$

is an exponentially monotone decreasing function of $u$. Furthermore, every solution of the equation (16) is less or equal to the solution of the equation

$$
\int_{0}^{\infty} e^{-u t}|W(t)| d t=1
$$

the solution of which will be negative if

$$
\int_{0}^{\infty}|W(t)| d t<1
$$

and positive if

$$
\int_{0}^{\infty}|W(t)| d t>1
$$

Hence, the result.
Note that if $W(t)>0$, then $W(t)=|W(t)|$.

Theorem 2.1(Chitnis, N. R., 2005). The disease-free equilibrium point, $x_{d f e}$, is locally asymptotically stable if $\mathrm{RO}<1$ and the rate constant of HBV is greater than the rate constant of nonspecific HBV removal $(\alpha>\lambda)$, and is unstable if either RO $>1$ or the rate constant of HBV is less than the rate constant of nonspecific HBV removal $(\alpha<\lambda)$

Proof: The eigenvalues of the Jacobian matrix $\mathrm{J}$ can be determined by solving the characteristic equation $|J-\lambda I|=0$. The reproduction ratio $\mathrm{RO}$ can be determined from the Jacobian matrix with the aid of the Routh-Hurwitz criteria.

The eigenvalues at the 'disease-free' equilibrium are given

$(-\lambda, \pi D-\varphi,-\tau, \vartheta D-\sigma,-\xi,-\sigma,-\omega,-\kappa,-\zeta, 0)$.

The Jacobian matrix for the linearization of the system (1)-(10) about the 'disease-free' admits eigenvalues that are all real and negative, and hence the fixed point is asymptotically stable. We find that, with the exception of a small number of cases, the system remains stable under perturbations of the parameters of the system.

Sensitivity analysis: Sensitivity analysis is used to determine how Isensitive" a model is to changes in the value of the parameters of the model and to changes in the structure of the model. In this paper, we focus on parameter sensitivity. Parameter sensitivity is usually performed as a series of tests in which the modeler sets different parameter values to see how a change in the parameter causes a change in the dynamic behavior of the system.

Sensitivity to interferon response: The parameters $\psi$ and $\beta$ characterize the interferon production rate constant and the rate constant of induction of resistant state in cells, respectively. If $\psi$ increased or lowered from its standard value, disease always develops for standard values of $\mathrm{V}(0)$ and $\mathrm{S}(0)$. However, when $\beta$ is high, the host remains asymptotic. The time of onset of disease does not depend on $\psi$ and $\beta$, but when either of these constants are higher, the duration of disease becomes shorter. Damage increases if either $\psi$ or $\beta$ is decreased. Very low values of $\psi$ or $\beta$ result in 
excessive damage (over 50\%) which may presumably lead to secondary infections or death (Iwasaki et al., 1977). When the interferon production rate constant is two times bigger than the baseline value (i.e., when $\psi=5,000$ ), then the host remains contagious for about 3-5 days. When this rate is two times less than the baseline (i.e., when $\psi=1,250$ ) then the contagious period is about $2-3$ days. So, the difference in the length of infectious period is significant for various levels of innate immune response.

Even in the absence of an innate response (when $\psi=0$ and $\beta=0$ ), the disease is eventually healed by the adaptive immune response and the organism will approach the healthy state.

Sensitivity to cellular component of innate immunity: The parameters ò and $\mu$ stand for the rate constant of production of effector cells and rate constant of removal of infected cells by effectors, respectively. For sufficiently large ò or $\mu$, the host is able to clear the disease without symptoms and typical disease conditions, given the standard initial immunity and standard initial amount of the virus. Although ò has no effect on the onset of the disease, when infected cell removal rate constant is high, the onset of infection happens later. At low values of ò or $\mu$, the symptoms last longer. When ò is high, the resulting maximum damage, is large and may result in death. On the other hand, when $\mu$ is high, we observe lower damage of cells. Even under a significant decrease in $\mu$, maximum damage will stay under $50 \%$ and hence a decrease in $\mu$ has no effect on the mortality. When there is a less vigorous cellular response, the host remains infectious for longer. But the time differences in the contagious period of the host for various levels of ò or $\mu$ are small.

Even in the absence of a cellular response (whenò $=0$ and $\mu=0$ ), virus is eventually cleared by the innate and adaptive immune responses and the organism will approach the healthy state.

Sensitivity to adaptive response: Activation of adaptive immune response is slower than activation of cellular and interferon components of innate immunity. The parameters $\phi, \tilde{\mathrm{n}}$ and $\varepsilon$ stand for the plasma cell production rate constant, antibody production rate constant and the rate constant of neutralization of HBV by antibodies. For sufficiently large $\phi, \tilde{\mathrm{n}}$ or $\varepsilon$, the host is able to clear infection without symptoms after administration of a standard inoculum. Although $\phi$ has no effect on the onset of the disease, later onset is observed with higher $\tilde{\mathrm{n}}$ and ". The duration of illness does not depend on $\phi$, $\tilde{\mathrm{n}}$ or $\varepsilon$. Damage is lower with higher $\%$, while damage is insensitive to the two other rate constants. Variations in $\phi, \tilde{n}$ or $\varepsilon$ never result in excessive damage. With higher $\tilde{\mathrm{n}}$, the contagious period is significantly shorter.

In summary, $\varepsilon$ only affects the onset of the disease, while $\phi$ affects only virus shedding at the peak. The system is much more sensitive to theñ .

\section{DISCUSSION}

We derived and analyzed a mathematical model to better understand the immune response to HBV infection. We used this model to compare strategies for HBV control for two representative areas which include innate and adaptive immunity.

Mathematically, we modeled the immune response to HBV as a 10 - dimensional system of ordinary differential equations. We first showed the existence of the equilibrium point without disease. The equilibrium point with no virus, $(0,1,0,0,0,0,0,0,0$, 0 ), is locally asymptotically stable if the rate constant of HBV, $\alpha$, is less than the rate constant of nonspecific HBV removal, $\lambda$.

Such a model could be used to explore in more detail individual determinants of symptoms and behavior of clinical relevance, especially in large-scale simulations of disease spread and containment. Simulation and sensitivity analysis of this model suggest that for majority of possible parameter values and initial conditions the course of the disease falls into one of three categories: asymptotic disease, typical disease, and severe disease. In special circumstances a recurrence of infection may occur, followed by a transition to either healthy or chronic state. The magnitude of initial viral load $V(0)$ determines which of the paths will be taken, in accordance with experimental observations (Tamura and Kutara, 2004). With sufficiently small viral inoculum, disease is asymptotic in the sense that the virus level decreases monotonically to 0 and damage remains very low.

Analysis of the adaptive immune response revealed that whenever there is sufficient antibody response 
with enough specificity, the dynamics will restore health, irrespective of the intensity of the innate responses and of the trajectory followed by the disease. However, if memory cells cannot produce sufficiently compatible antibodies against the HBV particles initially (very low $\mathrm{S}(0)$ ) or cannot improve the antigenic compatibility sufficiently rapidly ( $S$ is not increasing during the disease), there is transition of the system to a chronic state.

\section{CONCLUSION}

Although each component of innate and adaptive immune response contributes to the recovery of HBV infection, the simulations suggest that, in the absence of one component of innate immunity, the remaining two defense mechanisms are sufficient for viral clearance. For example, when cellular component are not involved in viral clearance, antibodies (sufficient in the amount and affinity) on their own can mediate clearance of hepatitis B (Scherle et al., 1992). The cellular response can also be sufficient to clear the HBV infection from an individual (Asquith et al., 2003). In the absence of adaptive immunity, the viral load is brought down to very low levels, al- though not completely cleared, which is supported by experiments showing that in some circumstances antibody response is necessary to clear the infection (Iwasaki et al.,1977). For example, in nude mice without antibodies the viral particles survive with chronic infection (Scherle et al., 1992). After transferring $\mathrm{T}$ helper cells into the infected mice that promotes antibody response, the disease is completely cleared (Iwasaki et al., 1977; Scherle et al., 1992). Therefore, one can conclude that innate defense mechanisms are not capable of curing the disease on their own in the absence of antibody response, and can only reduce the disease to a chronic state.

\section{REFERENCES}

Ada, G.L.,.Jones, P.D., (1986). The immune response to influenza infection. Curr. Top. Microbiol. Immunol.,128, 154.

Asquith B., Bangham C. R.,(2003). An introduction to lymphocyte and viral dynamics: the power and limitations of mathematical analysis. Proc. Biol. Sci.,720: 1651-1657.

Baris H., (2007) Mathematical Modeling Of Virus Dynamics In Immunology. J. Immunol., 144 , 8 - 19.

Barnaba V., Franco A., Alberti A., Balsano C., Benvenuto R., Balsano F.,(1989). Recognition of hepatitis B virus envelope proteins by liver- infiltrating $T$ lymphocytes in chronic HBV infection. J. Immunol., 143 , 2650-2655.

Bocharov, G.A.,.Romanyukha, A.A., (1994). Mathematical model of antiviral immune response.III. Inuenza $A$ virus infection. J. Theor. Biol., 167, 323-360

Chitnis N. R. (2005). Using mathematical models in controlling the Spread of malaria. 34-36

Ferrari C., Penna A., Giuberti T. (1987). Intrahepatic, nucleocapsid antigen-speci_c $T$ cells in chronic active hepatitis B. J. Immunol.,139 , 2050-2058.
Ganem D.,(1982) Persistent infection of humans with hepatitis $B$ virus: mechanisms and consequences. Rev. Infect. Dis., 4 , 1026-1047.

Guidotti L.G., Rochford R., Chung J., Shapiro M., Purcell R., Chisari F.V.(1999) Viral clearance without destruction of infected cells during acute HBV infection. Science, 284 , 825-829.

Guidotti L.G., Ando K., Hobbs M.V.,(1994). Cytotoxic T lymphocytes inhibit hepatitis $B$ virus gene expression by a noncytolytic mechanism in transgenic mice. Proc. Natl. Acad. Sci. USA, 91 , 3764-3768.

Guidotti L.G., Chisari F.V.,(2000). Cytokine-mediated control of viral infections. Virology,273 , 221-227.

Heise T., Guidotti L.G., Cavanaugh V.J., Chisari F.V.,(1999). Hepatitis B virus RNA-binding proteins associated with cytokine-induced clearance of viral RNA from the liver of transgenic mice. J. Virol., 73 , 474-481.

Hayden, F.G., Fritz, R., Lobo, M.C., Alvord, W., Strober, W., Straus, S.E., (1998). Local and systemic cytokine responses during experimental human inuenza $A$ virus infection. Relation to symptom formation and host defense. J. Clin. Invest,101, 643-649.

Iwasaki, T.,.Nozima, T., 1977. Defense mechanisms against primary influenza virus infection in mice. I. The roles of interferon and neutralizing antibodies and thymus dependence of interferon and antibody production. J. Immunol., 118, 256-263.

Livingston B.D., Crimi C., Grey H. (1997). The hepatitis B virus specific CTL responses induced in humans by lipopeptide vaccination are comparable to those elicited by acute viral infection. J. Immunol.,159, 1383-1392.

Maini M.K., Boni C., Lee C.K. (2000). The role of virus-specific CD8(+) cells in liver damage and viral control during persistent hepatitis B virus infection. J. Exp. Med., 191, 1269-1280.

Maini M.K., Reignat S., Boni C., Ogg G.S., King A.S., Malacarne F., (2000). T cell receptor usage of virusspecific CD8 cells and recognition of viral mutations during acute and persistent hepatitis B virus infection. Eur. J. Immunol, 30: 3067 -3078.

Marchuk, G.I., Petrov, R.V., Romanyukha, A.A., Bocharov, G.A., (1991). Mathematical model of antiviral immune response. I. Data analysis, generalized picture construction and parameters evaluation for hepatitis B. J. Theor. Biol.,151 , 1-40.

Rehermann B.,(2000). Intrahepatic T cells in hepatitis B: viral control versus liver cell injury. J. Exp. Med.,191, 12631268.

Rehermann B., Lau D., Hoofnagle J.H., Chisari F.V.,(1996). Cytotoxic $T$ lymphocyte responsiveness after resolution of chronic hepatitis B virus infection. J. Clin. Invest.,97, 1655-1665.

Scherle, P.A., Palladino, G., Gerhard, W., (1992). Mice can recover from pulmonary inuenza virus infection in the absence of class I-restricted cytotoxic T cells. J. Immunol., 148, 212-217.

Zdanor, V. M., Bakrinskaja, A. G., (1969). Myxovimises Reproduction. Medicina. 